\title{
Análise do produto memoryn life da life natural
}

\section{Dr. Fabiano de Abreu Agrela Rodrigues ${ }^{1}$}

deabreu.fabiano@gmail.com

\section{RESUMO}

A memória é a capacidade em que o nosso cérebro possui de adquirir, armazenar e recuperar informações disponíveis, de modo interno no cérebro. É o armazenamento de informações e fatos obtidos através de experiências ouvidas ou vividas. Diante dos prejuízos que possam ocorrer com o passar da idade e devido a esta cultura atual da sociedade, foram criados suplementos no mercado com objetivo de melhorar o armazenamento da memória. Assim, o presente estudo pretende avaliar os efeitos do produto Memoryn da empresa Life Natural ${ }^{\circledR}$ a partir de uma revisão de literatura realizada por meio das seguintes bases de dados: PubMed, Scielo e Google Acadêmico, através da análise da composição do produto, usando como referência, os dados enviados pela empresa Life Natural, cujo produto é aprovado e assinado pelo neurocientista, Doutor Fabiano de Abreu Rodrigues.

Palavras-chave: memória; suplementação; neurociencia; cérebro; função cognitiva.

\footnotetext{
${ }^{1} \mathrm{PhD}$, neurocientista, mestre em psicanálise, biólogo, historiador, antropólogo, com formações também em neuropsicologia, neurolinguística, neuroplasticidade, inteligência artificial, neurociência aplicada à aprendizagem, filosofia, jornalismo, programação em python e formação profissional em nutrição clínica Diretor do Centro de Pesquisas e Análises Heráclito; Chefe do Departamento de Ciências e Tecnologia da Logos University International e da City University; Membro da SFN - Society for Neuroscience, Membro Redilat.
} 


\title{
Life natural memoryn life product review
}

\begin{abstract}
Memory is our brain's ability to acquire, store and retrieve available information, internally in the brain. It is the storage of information and facts obtained through experiences heard or lived. Given the damage that may occur with age, supplements were created on the market with the aim of improving memory storage. Thus, the present study intends to evaluate the effects of the Memoryn product from the company Life Natural ${ }^{\circledR}$ from a literature review carried out through the following databases: PubMed, Scielo and Google Academic, through the analysis of the composition of the product, using as reference, the data sent by the company Life Natural, whose product is approved and signed by the psychologist, Dr. Fabiano de Abreu.
\end{abstract}

Keywords: memory; supplementation; neuroscience; brain; cognitive function.

Artículo recibido: 02 enero 2022 Aceptado para publicación: 28 enero 2022 Correspondencia: deabreu.fabiano@gmail.com Conflictos de Interés: Ninguna que declarar 


\section{INTRODUÇÃO}

\section{Memória}

A memória é a habilidade que o cérebro tem de armazenar informações de maneira que podemos recuperá-las diante da necessidade. Acontece por meio da formação de conexões das células nervosas no cérebro. Tais conexões são ligadas por pontos chamados sinapses (Lee, 2017).

No momento em que captamos informações por meio dos órgãos visuais elas são encaminhadas para partes distintas do córtex visual e o córtex frontal recebe as informações operacionais de curto prazo como forma de utilização imediata. Essas informações são encaminhadas para o hipocampo que é responsável pela conversão em armazenamento de longo prazo utilizando-se de fatores emocionais vinculados para que essas possam ser reativadas quando necessárias (Abadie, 2019; Bergstrom, 2016).

Alguns fatores emocionais podem auxiliar no processo de memorização de onde são formados através de conexões neurais localizadas na amígdala permitindo a ação rápida. Na região do cerebelo e nos gânglios basais são memorizados as habilidades e hábitos inconscientes como por exemplo andar, dirigir e outros (Zhang, 2020).

Algumas doenças neurológicas, distúrbios psicológicos, problemas metabólicos, intoxicações, álcool, drogas, medicamentos, fumo e cafeína podem influenciar de maneira negativa no processo de memorização comprometendo-a e, de maneira natural, o córtex cerebral vai se desgastando, suas atividades serão reduzidas e tais fatores podem acelerar o processo e torná-lo precoce (Marshall, 2006).

É possível melhorar o funcionamento do córtex, através de uma alimentação saudável, boas noites de sono, técnicas mentais como por exemplo exercícios de relaxamento, associação de fatos a imagens, leituras em voz alta, anotar fatos que julgar importantes, anotar ideias entre outras (Brainerd, 2018; Phelps, 2019).

A memória divide-se em 3 categorias: sensorial, atua para reter as informações que nos chegam pelos sentidos (visão, audição, tato, olfato e paladar). Ela é processada, analisada, interpretada e guardada no cérebro em menos de 2 segundo como se fosse uma memória imediata (Roozendaal, 2011).

A memória de curta duração, é usada quando o cérebro entende que a informação recebida é importante: a informação é transferida da memória sensorial para a memória de curta duração, podendo ser memorizada em até 7 informações durante cerca de 30 
segundos. Se for necessário é armazenada durante um período maior e para isso, o cérebro recorre ao último tipo de memória (Robertson, 2002).

A memória de longa duração, ocorre quando a memória ultrapassa os primeiros dois tipos e a informação pode ser guardada no espaço de longa duração. Esta é a parte da memória mais interessante que nos permite guardar as informações de várias formas sempre de uma maneira cronológica, já que é o tipo de organização a que estamos habituados (Born, 2012).

Existem também 3 categorias de memória; semântica, que é quando o cérebro guarda o que está armazenado na memória de longa duração e formula as nossas ideias, os conceitos e os significados (Ackermann, 2014; de Quervain, 2017).

Memória de Processo: parte da memória de longa duração, que é guardada as informações sobre os processos cotidianos com execução espontânea como andar, falar, dirigir, usar o computador e outros (Ackermann, 2014; de Quervain, 2017).

Memória Esporádica: parte da memória de longa duração, que nos possibilita a recuperação de episódios vividos no passado (Ackermann, 2014; de Quervain, 2017).

O processo de nutrição é multifatorial e há uma preocupação em como impacta no cérebro sem excessos quanto a necessidade do uso da suplementação. O estilo de vida atual compromete a homeostase do organismo, levando a uma necessidade de suplementação caso necessário em prol do bem -estar do indivíduo (Rodrigues, Fabiano de Abreu, 2021).

\section{METODOLOGIA}

O presente estudo se trata de uma revisão de literatura realizada por meio das seguintes bases de dados: PubMed, Scielo e Google Acadêmico por meio das seguintes palavras chaves: memória, suplementação, neurociência, cérebro e função cognitiva e partir dos compostos dispostos na bula do produto e das informações passadas pelo fabricante: Life Natural ${ }^{\circledR}$. Buscamos orientar a pesquisa analisando sistematicamente cada composto do produto e sua ligação com a função cognitiva: memória.

\section{Ativos para melhora da memória}

Fosfatidilserina é um componente fosfolipídico geralmente mantido no folheto interno, ao lado do citosólico das membranas celulares, através de uma enzima denominada translocase aminofosfolipídeo ATP. Quando uma célula passa por morte por apoptose, a fosfatidilserina não está mais restrita ao lado citosólico da membrana e torna-se exposta por meio superfície da célula (Glade, 2015; Kim, 2014). 
Derivada de um aminoácido é encontrada em enormes quantidades no cérebro no tecido neural fazendo parte da membrana celular e pode contribuir para a função cognitiva, principalmente dos idosos, melhorando a memória e a atenção (Kim, 2014).

O composto é gerado pelo organismo podendo também ser obtido por meio da alimentação em carnes vermelhas, frango, peru e peixe principalmente nas vísceras, como fígado ou rins e por meio da suplementação (Sharma, 2018).

Dentre os seus benefícios destaca-se: melhorar a função cognitiva e a memória de indivíduos portadores de Alzheimer e indivíduos com comprometimento da memória associado à idade, pois, atua na prevenção ou redução da deficiência cognitiva e demência (Nagata, 2020).

A fosfatidilserina atua no aumento da comunicação neuronal aumentando a fluidez das membranas celulares e os níveis de acetilcolina, que é um importante neurotransmissor. Age na proteção das membranas celulares e nos danos oxidativos e dos radicais livres (Nagata, 2020).

Figura 1 - Estrutura química da fosfatidilserina

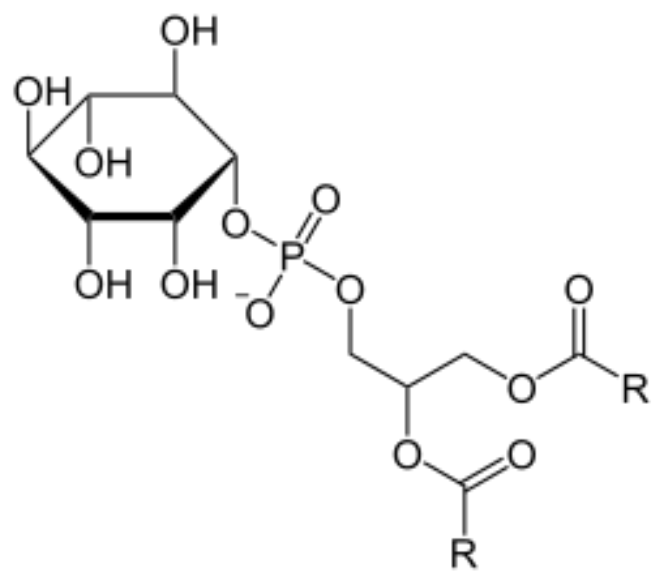

Fonte: Adaptado de Antonsson et al. 1997

Óleo de peixe é derivado dos tecidos de peixes oleosos. Contêm os ácidos graxos ômega3, ácido eicosapentaenóico (EPA) e ácido docosahexaenóico (DHA), precursores de alguns eicosanóides que possuem função de reduzir a inflamação no corpo e na melhora da hipertrigliceridemia (Burhani, 2017; Gao, 2017).

O Ômega 3 atua na formação da bainha de mielina, que é um componente dos neurônios e é responsável pela manutenção do sistema nervoso central. Sendo a principal substância em sua composição, o ácido alfa linolênico que age na produção dos ácidos, 
eicosapentaenoico (EPA) e docosahexaenoico (DHA) no organismo, atuam prol da redução de triglicérides, que é um tipo de gordura, que quando em níveis altos, prejudica a saúde. Também age no aumento do colesterol (HDL) conhecido como colesterol bom (Burhani, 2017; Gao, 2017).

Figura 2 - Estrutura química do óleo de peixe (Ômega 3)

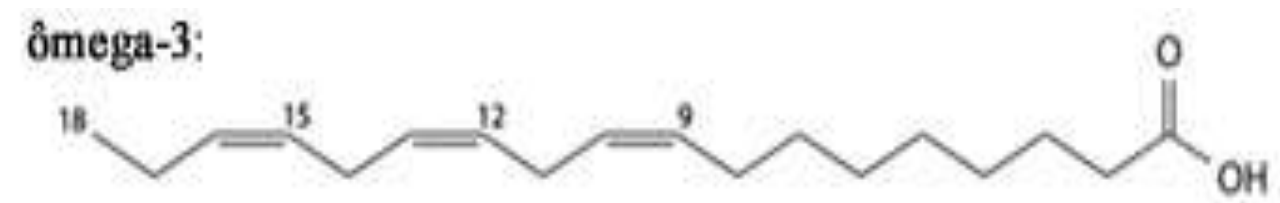

Fonte: Adaptado de Fogaça et al. 2019

Óleo de girassol é extraído da semente da planta de mesmo nome e é usado como fonte de alimento, como biocombustível e no preparo de alimentos (Summers, 2019).

É gorduroso e rico em ácidos graxos poli e monoinsaturados $(62 \%$ e $25 \%$ de sua composição, respectivamente) destacando o ômega 9. Rico em vitamina E, poderoso antioxidante. Em uma colher de sopa $(12 \mathrm{ml})$ possui 4,92 $\mathrm{mg}$ da vitamina (Summers, 2019; Rele, 2003). É utilizado em cosméticos devido aos altos níveis de concentração dessa vitamina.

No uso tópico atua para proteção e fortalecimento. Age na prevenção do envelhecimento precoce acelerado, mantém a pele hidratada, macia e com vitalidade; pode ser usado na limpeza da pele em casos de acne; age como um poderoso anti-inflamatório auxiliando no processo de cicatrização de queimaduras, cicatrizes antigas e queimaduras de segundo e terceiro grau. Possui propriedades que atuam na proteção contra os raios UV e, por isso, é usado na composição de protetores solares naturais; pode ser utilizado em peles de bebês, puro ou como carreador para óleos essenciais (Walters, 2019).

Figura 3 - Estrutura química do óleo de girassol

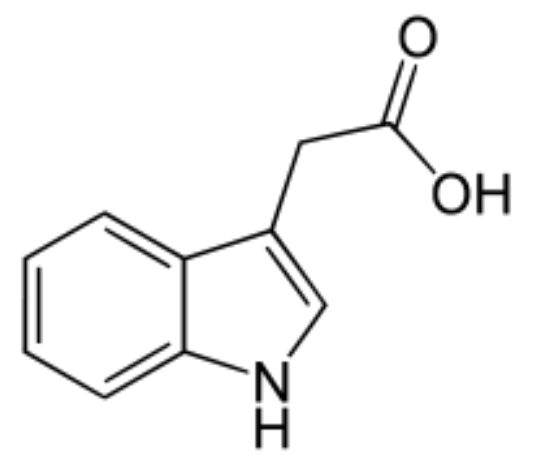

Fonte: Adaptado de Amaral et al. 2018 
Lecitina de Soja é um suplemento obtido por meio da extração do óleo dos grãos da soja, rico em vitamina B8 (colina), fósforo e demais compostos que promovem a saúde e o bem-estar (Hirose, 2018). Composta por glicolípidos, triglicéridos e fosfolípidos ${ }^{2}$.

Na bioquímica, o termo lecitina é utilizado como sinônimo de fosfatidilcolina pura, um fosfolípido que constitui o principal componente da fracção fosfatada que se obtém da gema de ovo ou de grãos de soja e girassol de onde é extraída por meios mecânicos ou químicos, utilizando hexano (Hirose, 2018; Lee, 2019).

Os benefícios da lecitina de soja se destacam na melhora da saúde da mulher, sintomas da TPM e alívio dos sintomas da menopausa, nas doenças psíquicas, como por exemplo como suplementação no tratamento de doenças como Alzheimer, estresse e na redução dos níveis sanguíneos de colesterol e triglicerídeos, prevenindo doenças cardiovasculares (Moré, 2014).

Figura 4 - Estrutura química da Lecitina de Soja

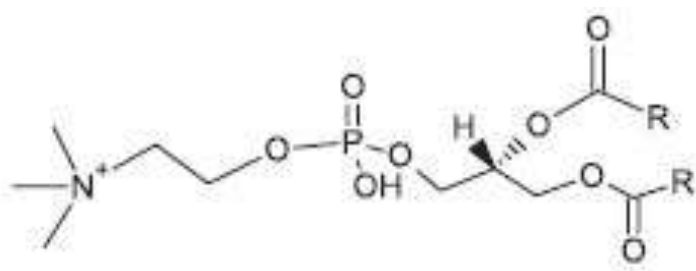

Fonte: Adaptado de Mertins et al. 2008

\section{Memoryn da Life Natural ${ }^{\circledR}$}

O produto Memoryn da empresa Life Natural ${ }^{\circledR}$, que utiliza componentes naturais atua na melhoria da memória, na concentração, motivação, habilidades motoras, velocidade de reação, neutralização do stress, ajudando a acelerar as respostas celebrais, aumentando a capacidade de leitura, diminuindo os problemas de comportamento, aumentando a capacidade mental além de auxiliar no tratamento do mal de Alzheimer.

Sua composição encapsulada dispõe de: gelatina, água purificada, umectante glicerina e corante cúrcuma. Seus principais ingredientes são: óleo de peixe concentrado, lecitina de soja, alto teor de fosfatidilserina, óleo de girassol, glaceante cera de abelha, emulsificante lecitina de girassol. Para obter um bom resultado, o fabricante recomenda a ingestão de 1 cápsula por dia.

A seguir a bula do produto contendo as informações passadas pelo fabricante:

\footnotetext{
${ }^{2}$ por exemplo: fosfatidilcolina, fosfatidiletanolamina e fosfatidilinositol.
} 
Tabela 1 - Tabela Nutricional do Memoryn da Life Natural ${ }^{\circledR}$

\begin{tabular}{|l|c|c|}
\hline TABELA NUTRICIONAL & QTD. /DOSE & \% VD* \\
\hline VALOR ENERGÉTICO & $37,4 \%$ & $2 \%$ \\
\hline Gorduras Totais & $0,0 \mathrm{~g}$ & $40 \%$ \\
\hline Vitamina B5 & $2,0 \mathrm{mg}$ & $83 \%$ \\
\hline Vitamina B1 & $1,0 \mathrm{mg}$ & $15 \%$ \\
\hline Fibra Alimentar & $3,8 \mathrm{mg}$ & $1,2 \%$ \\
\hline Proteínas & $0,6 \mathrm{mg}$ & \\
\hline
\end{tabular}

*Não contém quantidades significativas de Carboidratos, Proteínas, Gorduras Totais, Gorduras Saturadas, Gorduras Trans e Sódio.

É indicado para indivíduos maiores de 19 anos e contraindicado para gestantes, lactantes e alérgicos pois, contém corante, soja, peixe e gelatina em sua fórmula.

\section{CONSIDERAÇÕES FINAIS}

Por meio da análise da composição do produto usado como referência e dos dados enviados pela empresa Life Natural, bem como a aprovação e assinatura do produto neurocientista, Doutor Fabiano de Abreu Rodrigues, é possível afirmar a eficácia do produto Memoryn, pois ele contém substâncias que possuem comprovação científica por meio da publicação na revista científica e no Centro de Pesquisas e Análises Heráclito CPAH Scientific Journal of Health confirmando que são eficientes para a melhora da memória.

\section{REFERÊNCIAS}

ABADIE, M., Camos, V. False memory at short and long term. J Exp Psychol Gen. v. 148, n. 8, págs. 1312-1334, 2019 doi:10.1037/xge0000526

ACKERMANN, S., Rasch, B. Differential effects of non-REM and REM sleep on memory consolidation? Curr Neurol Neurosci Rep. v. 14, n. 2, 2014. doi:10.1007/s11910-013-0430-8

AMARAL, M. Por que o girassol "acompanha" o movimento do sol? Médium. 2018 
ANTONSSON, B. Fosfatidilinositol sintase de tecidos de mamíferos. Biochimica e Biophysica Acta, 1997

BERGSTROM, H.C. The neurocircuitry of remote cued fear memory. Neurosci Biobehav Rev. v. 71, págs. 409-417,2016 doi:10.1016/j.neubiorev.2016.09.028

BORN, J., Wilhelm, I. System consolidation of memory during sleep. Psychol Res. v. 76, n. 2, págs. 192-203, 2012 doi:10.1007/s00426-011-0335-6

BRAINERD, C.J., Reyna, V.F. Complementarity in false memory illusions. J Exp Psychol Gen. v.147, n. 3, págs. 305-327, 2018. doi:10.1037/xge0000381

BURHANI, M.D., Rasenick, M.M. Fish oil and depression: The skinny on fats. J Integr Neurosci. v. 16, págs. 115-124, 2017 doi: 10.3233/JIN-170072.

DE QUERVAIN, D., Schwabe, L., Roozendaal, B. Stress, glucocorticoids and memory: implications for treating fear-related disorders. Nat Rev Neurosci. 2017 v. 18, n. 1, págs. 7-19, 2017 doi:10.1038/nrn.2016.155

FOGAÇA, J.R.V. Exercícios Sobre Isomeria Plana. Mundo. Educação, 2019

GAO H, Geng T, Huang T, Zhao Q. Fish oil supplementation and insulin sensitivity: a systematic review and meta-analysis. Lipids Health Dis. v. 3, n. 16, e1, 2017 doi: 10.1186/s12944-017-0528-0.

GLADE, M.J., Smith, K. Phosphatidylserine and the human brain. Nutrition. v. 31, n. 6, págs. 781-6, 2015 doi: 10.1016/j.nut.2014.10.014.

HIROSE, A., Terauchi, M., Osaka, Y., Akiyoshi, M., Kato, K., Miyasaka, N. Effect of soy lecithin on fatigue and menopausal symptoms in middle-aged women: a randomized, double-blind, placebo-controlled study. Nutr J. v. 8, n. 17, 2018. doi: 10.1186/s12937-018-0314-5.

KIM, H.Y., Huang, B.X., Spector, A.A. Phosphatidylserine in the brain: metabolism and function. Prog Lipid Res. v. 56, págs. 1-18, 2014 doi: 10.1016/j.plipres.2014.06.002.

LE, N.T.T., Cao, V.D., Nguyen, T.N.Q., Le, T.T.H., Tran, T.T., Hoang, T.T. Soy Lecithin-Derived Liposomal Delivery Systems: Surface Modification and Current Applications. Int J Mol Sci. v. 23, n. 20, e4706, 2019 doi: 10.3390/ijms20194706.

LEE, J.L.C., Nader, K., Schiller, D. An Update on Memory Reconsolidation Updating. Trends Cogn Sci. v. 21, n. 7, págs. 531-545, 2017 doi: 10.1016/j.tics.2017.04.006. 
MARSHALL, L., Helgadóttir, H., Mölle, M., Born, J. Boosting slow oscillations during sleep potentiates memory. Nature. v. 444, n. 7119, págs. 610-613, 2006 doi:10.1038/nature05278

MERTINS, O., Sebben, M., Schneider, P.H., Pohlmann, A.R. Caracterização da pureza de fosfatidilcolina da soja através de $\mathrm{RMN}^{\mathrm{de}}{ }^{1} \mathrm{H}$ e de 31P. Nota técnica. Quím. Nova v. 31, n. 7, 2008

MORÉ, M.I., Freitas, U., Rutenberg, D. Positive effects of soy lecithin-derived phosphatidylserine plus phosphatidic acid on memory, cognition, daily functioning, and mood in elderly patients with Alzheimer's disease and dementia. Adv Ther. v. 31, n. 12, págs. 1247-62, 2014 doi: 10.1007/s 12325-0140165-1.

NAGATA, S., Sakuragi, T., Segawa, K. Flippase and scramblase for phosphatidylserine exposure. Curr Opin Immunol. v. 62, págs. 31-38, 2020 doi: 10.1016/j.coi.2019.11.009.

PHELPS, E.A., Hofmann, S.G. Memory editing from science fiction to clinical practice. Nature. 2019 v. 572, n. 7767, págs. 43-50, 2019 doi:10.1038/s41586019-1433-7

RELE, A.S., Mohile, R.B. Effect of mineral oil, sunflower oil, and coconut oil on prevention of hair damage. J Cosmet Sci. v. 54, n. 2, págs. 175-92, 2003

RODRIGUES, Fabiano de Abreu. Nutrition process for better memorization. CPAH Scientific Journal of Health. V. 1, n. 3, págs. 139-145, 2021.

ROBERTSON, L.T. Memory and the brain. J Dent Educ. v. 66, n. 1, págs. 30-42, 2002 ROOZENDAAL, B., McGaugh, J.L. Memory modulation. Behav Neurosci. v. 125, n. 6, págs. 797-824, 2011 doi:10.1037/a0026187

SHARMA, B., Kanwar, S.S. Phosphatidylserine: A cancer cell targeting biomarker. Semin Cancer Biol. v. 52, n. 1, págs. 17-25, 2018 doi: 10.1016/j.semcancer.2017.08.012.

SUMMERS, A., Visscher, M.O., Khatry, S.K., Sherchand, J.B., LeClerq, S.C., Katz, J., Tielsch, J.M., Mullany, L.C. Impact of sunflower seed oil versus mustard seed oil on skin barrier function in newborns: a community-based, clusterrandomized trial. BMC Pediatr. v. 23, n. 19, e1, 2019 doi: 10.1186/s12887-0191871-2. 
WALTERS, D., Ndau, E., Saleh, N., Mosha, T., Horton, S. Cost-effectiveness of sunflower oil fortification with vitamin A in Tanzania by scale. Matern Child Nutr. v.15, n. 3, e12720, 2019 doi: 10.1111/mcn.12720.

ZHANG, J., Yetton, B., Whitehurst, L.N., Naji, M., Mednick, S.C. The effect of zolpidem on memory consolidation over a night of sleep. Sleep. v. 43, n. 11, 2020 doi:10.1093/sleep/zsaa084 